\title{
Object Recognition with Lower Order Gegenbauer Moments
}

\author{
Simon Liao and Jing Chen
}

\begin{abstract}
Moment descriptors have been used as the image features in pattern recognition since the early development stage of moment method. The moment method is different from other object feature extraction methods as it captures global properties of an object rather than the local ones. In this research, an effective procedure to select moment features of an object is proposed. By using a set of 6,763 Chinese characters as the testing objects, we will show that the new Gegenbauer moment features perform very well in recognizing all Chinese characters including those that are very close in shapes.
\end{abstract}

Index Terms-Moment descriptors, gegenbauer moments, object recognition, Chinese character recognition.

\section{INTRODUCTION}

Various types of moment descriptors have been developed following $\mathrm{Hu}[1]$ as the extracted features of an image in pattern recognition [2]. The moment-based features are different from many other features used in object recognition because the moment method captures global properties of images rather than the local compositions. Moments of an image can represent the image in the moment space uniquely and this uniqueness nature of moments makes the method of moments an appropriate candidate in object recognition, such as Chinese character recognition [3], [4]. Also, some moment functions are invariant under translation, scale change, and rotation, which are essential features required by many object recognition systems.

The recognition rate of an object recognition system largely depends on the capacities of the extracted features to distinguish one object from another. Generally speaking, the more features a system maintains, the higher the correction rate of the recognition system will be. However, some large object sets only allow a small number of features to be used in order to perform the recognition tasks faster. Therefore, the method of selecting more efficient features becomes the single most important factor in achieving high recognition rate for many object recognition systems.

In this research, we will introduce a new object recognition model with moment descriptors. From statistics perspective, we select four lower order moments with the highest variance values to form a set of moment features in the four dimensional Gegenbauer moment space. To test this new object recognition system, we used a set of 6,763 Chinese characters, which are defined in the Chinese standard

Manuscript received May 11, 2013; revised July 30, 2013.

Simon Liao and Jing Chen are with the University of Winnipeg, Winnipeg, Manitoba, Canada (e-mail: s.liao@uwinnipeg.ca, je.chen@uwinnipeg.ca).
GB2312, as the testing objects. The experiment results show that our Gegenbauer moment features not only perform very well in terms of classifying all the testing Chinese characters, they perform particularly well in recognizing Chinese characters that are very close in shapes. Also, by changing the parameter $\lambda$ values of the Gegenbauer polynomials, the same set of features can perform as various types of moment features and enhance the recognition power.

Although the new moment-based object recognition model is only tested by applying a set of Chinese characters, it is expected to be able to provide similar recognition power to other sets of image objects. Especially, this proposed object recognition system based on the Gegenbauer moment features is an ideal complement for other object recognition techniques depended on the local structures of an object.

The rest of the paper is organized as follows. Section II generally describes some basic moment properties. Section III introduces the Gegenbauer polynomials and moments, which will be applied in this research. Section IV presents and discusses the experiment results. Section V represents our observations and conclusions.

\section{OBJeCt RECOGNITION VIA MOMENTS}

The use of moment descriptors of various forms as features of an object for image analysis and pattern recognition was inspired by $\mathrm{Hu}$ [1]. Hu stated that if $f(x, y)$ is piecewise continuous and has nonzero values in a finite region of the $(x$, y) plane, then the geometric moment sequence.

$$
M_{p q}=\int_{a_{1}}^{a_{2}} \int_{b_{1}}^{b_{2}} x^{p} y^{q} f(x, y) d x d y
$$

is uniquely determined by $f(x, y)$, and conversely, $f(x, y)$ is uniquely determined by the moment sequence $\left\{M_{p q}\right\}$.

The lower order geometric moments represent some well known physical properties of the underlying image function. The definition of the zeroth order geometric moment, $M_{00}$, of the function $f(x, y)$

$$
M_{00}=\int_{a_{1}}^{a_{2}} \int_{b_{1}}^{b_{2}} f(x, y) d x d y
$$

represents the total mass of the given image function $f(x, y)$. When computed for a binary image, the zeroth moment (2) gives the total area of the image.

The two first order geometric moments,

$$
M_{10}=\int_{a_{1}}^{a_{2}} \int_{b_{1}}^{b_{2}} x f(x, y) d x d y
$$




$$
M_{01}=\int_{a_{1}}^{a_{2}} \int_{b_{1}}^{b_{2}} y f(x, y) d x d y
$$

represent the centre of mass of the image function $f(x, y)$. The centre of mass is the point where all the mass of the image could be concentrated without changing the first moment of the image about any axis. In terms of moment values, the coordinates of the centre of mass are

$$
\bar{x}=\frac{M_{10}}{M_{00}}, \quad \bar{y}=\frac{M_{01}}{M_{00}}
$$

The centre of mass can be chosen to represent the position of an image in the field of view. The equations in (5) define a unique location of the image $f(x, y)$ that can be used as a reference point to describe the position of the image.

The second order moments, $\left\{M_{02}, M_{11}, \mathrm{M}_{20}\right\}$ known as the moments of inertia, may be used to determine the orientation of image $f(x, y)$, an important image feature. In general, the orientation of an image describes how the image lies in the field of view, or the directions of the principal axes. For example, $M_{02}$ and $M_{20}$ are the moments of inertia of $f(x$, $y$ ) around the $x$ and $y$ axes, respectively.

An alternative means of describing image properties represented by moments is to consider the relationship between the moments of an image and those of the projections of that image. The moments in the sets $\left\{M_{p 0}\right\}$ and $\left\{M_{0 p}\right\}$ are equivalent to the moments of the image projection onto the $x$ axis and $y$ axis, respectively.

Describing an image function $f(x, y)$ with moments means that the global properties of the image are used rather than the local ones. $\mathrm{Hu}$ [1] first demonstrated the utility of moment features through a simple pattern recognition experiment. The experiment was performed by using a set of 26 capital English letters as input patterns.

The lower order moments, i.e., order 0 to 3 , are effective features in object recognition systems. For example, various forms of moment features have been applied in Chinese character recognition systems with decent results.

\section{GEGENBAUER MOMENTS}

The Gegenbauer polynomial, which is also called Ultraspherical polynomial, is a class of orthogonal polynomials on the interval $[-1,1]$ characterized by a parameter $\lambda$ that can change the "form" of the polynomial. We use $P_{n}^{(\lambda)}(x)$ to denote the Gegenbauer polynomial of order $n$ with the parameter $\lambda$, which can be any real number satisfying the restriction of $\lambda>-0.5$ [5], [6].

The Gegenbauer polynomial is a special case of Jacobi polynomial $P_{n}^{(\alpha, \beta)}(x)$ with $\alpha=\beta$, and can be expressed in the notation

$$
P_{n}^{(\lambda)}(x)=\frac{\Gamma(\lambda+1 / 2)}{\Gamma(2 \lambda)} \frac{\Gamma(\mathrm{n}+2 \lambda)}{\Gamma(\mathrm{n}+\lambda+1 / 2)} P_{n}^{(\lambda-1 / 2, \lambda-1 / 2)}(x),
$$

where $\Gamma(x)$ is the Gamma function.
When $\lambda=0.5$, the Gegenbauer polynomial $P_{n}^{(\lambda)}(x)$ becomes the Legendre polynomial $P_{n}(x)$.

Let $f(x)$ be a function defined on $[-1,1]$ such that

$$
\int_{-1}^{1} f^{2}(x)\left(1-x^{2}\right)^{\lambda-1 / 2} d x<\infty
$$

then $f(x)$ can be represented by the orthogonal expression respecting to $P_{n}^{(\lambda)}(x)$,

$$
f(x)=\sum_{n=0}^{\infty} A_{n}(\lambda) P_{n}^{(\lambda)}(x),
$$

where $A_{n}(\lambda)$ is the Gegenbauer moment and given by

$$
A_{n}(\lambda)=C_{n}(\lambda) \int_{-1}^{1} f(t) P_{n}^{(\lambda)}(t)\left(1-t^{2}\right)^{\lambda-1 / 2} d t
$$

$C_{n}(\lambda)$ is the normalizing constant

$$
C_{n}(\lambda)=\frac{2^{2 \lambda} \Gamma^{2}(\lambda)}{2 \pi} \frac{n !}{\Gamma(n+2 \lambda)}(n+\lambda), \lambda \neq 0 .
$$

For an image function $f(x, y)$, we can define the Gegenbauer moment in a two-dimensional space as

$$
\begin{aligned}
& A_{p q}(\lambda)=C_{p}(\lambda) C_{q}(\lambda) \\
& \int_{-1}^{1} \int_{-1}^{1} f(u, v) P_{p}^{(\lambda)}(u) P_{q}^{(\lambda)}(v)\left(1-u^{2}\right)^{\lambda-1 / 2}\left(1-v^{2}\right)^{\lambda-1 / 2} d u d v
\end{aligned}
$$

with $\lambda>-0.5$.

Different combinations of lower order moment features have been applied in object recognition with decent results [3], [4].

In order to extract more efficient lower order moment features that can differentiate objects more effectively, we conduct the data analysis. While the mean of a moment represents the average magnitude of an object, the variance of the moment measures the dispersion of its distribution. From statistics perspective, the moment with a higher variance can be the more effective feature to differentiate different objects than those with the lower variances. With using a set of Chinese characters as the testing objects, among all of ten lower order moments, we found that it is an effective way for object recognition by simply choosing four moments with the highest variance values to form four moment function features $f_{1}, f_{2}, f_{3}$, and $f_{4}$.

To test our moment function features, we use the set of 6,763 Chinese characters defined in China's national standard GB2312, with the font of song, as the testing objects. All of these Chinese characters are binary images and are composed of $24 \times 24$ pixels. Compared with other sets of objects, such as the set of English letters, the set of Chinese characters is larger and more difficult to classify. Since many Chinese characters with very different meanings are very similar in shapes, the Chinese character recognition is a challenging task in the field of object recognition. Although all of testing objects are binary images, there is no limitation for using the moment features on multi-gray-level images. All results of 
this research can be extended to the multi-gray-level image objects.

\section{EXPERIMENTAL RESULTS}

As mentioned in Section III, by changing the value of parameter $\lambda$, the "form" of Gegenbauer moments will be changed. In other words, by applying a set of Gegenbauer moment function features with different $\lambda$ values to the same object, we will obtain different results because the features are based on different types of moments.

First, we calculated all of the lower order, i.e., 0 to 3, Gegenbauer moments variance values of 6,763 Chinese characters with different parameter $\lambda$ values from 0.1 to 2.0 . The results are shown in Table I (A) and Table I (B).

TABLE I (A): VARIANCE VALUES OF FIVE LOWER ORDER GEGENBAUER MOMENTS, $A_{00}, A_{01} \mathrm{~A}_{10}, A_{02}$ AND $A_{11}$ WITH DIFFERENT $\lambda S$

\begin{tabular}{cccccc}
\hline \hline$\lambda$ & $A_{00}$ & $A_{01}$ & $A_{10}$ & $A_{02}$ & $A_{11}$ \\
\hline 0.1 & .00164 & .01855 & .01528 & .10250 & .47494 \\
0.2 & .00199 & .00597 & .00511 & .02513 & .04178 \\
0.3 & .00231 & .00323 & .00287 & .01086 & .01090 \\
0.4 & .00259 & .00212 & .00196 & .00591 & .00434 \\
0.5 & .00282 & .00154 & .00148 & .00365 & .00216 \\
0.6 & .00303 & .00119 & .00118 & .00244 & .00123 \\
0.7 & .00320 & .00096 & .00098 & .00172 & .00077 \\
0.8 & .00335 & .00079 & .00083 & .00127 & .00051 \\
0.9 & .00349 & .00067 & .00072 & .00096 & .00036 \\
1.0 & .00360 & .00057 & .00026 & .00074 & .00026 \\
1.1 & .00370 & .00050 & .00057 & .00059 & .00020 \\
1.2 & .00379 & .00044 & .00051 & .00047 & .00015 \\
1.3 & .00387 & .00039 & .00047 & .00039 & .00012 \\
1.4 & .00394 & .00035 & .00043 & .00032 & .00010 \\
1.5 & .00400 & .00032 & .00039 & .00027 & .00008 \\
1.6 & .00406 & .00030 & .00037 & .00023 & .00007 \\
1.7 & .00412 & .00027 & .00034 & .00019 & .00006 \\
1.8 & .00417 & .00025 & .00032 & .00017 & .00005 \\
1.9 & .00422 & .00024 & .00030 & .00015 & .00004 \\
2.0 & .00427 & .00022 & .00029 & .00013 & .00004 \\
\hline \hline
\end{tabular}

TABLE I (B): VARIANCE VALUES OF FIVE LOWER ORDER GEGENBAUER MOMENTS, $A_{20}, A_{03}, A_{12}, A_{21}$, AND $A_{30}$ WITH DIFFERENT $\lambda S$

\begin{tabular}{cccccc}
\hline \hline$\lambda$ & $A_{20}$ & $A_{03}$ & $A_{12}$ & $A_{21}$ & $A_{30}$ \\
\hline 0.1 & .08546 & .08536 & 1.8452 & 1.8107 & .12070 \\
0.2 & .02121 & .01961 & .13414 & .12372 & .02708 \\
0.3 & .00950 & .00803 & .02972 & .02623 & .01089 \\
0.4 & .00545 & .00418 & .01026 & .00881 & .00561 \\
0.5 & .00358 & .00249 & .00448 & .00380 & .00332 \\
0.6 & .00256 & .00161 & .00226 & .00192 & .00216 \\
0.7 & .00194 & .00111 & .00126 & .00109 & .00150 \\
0.8 & .00152 & .00080 & .00075 & .00067 & .00110 \\
0.9 & .00123 & .00059 & .00047 & .00043 & .00083 \\
1.0 & .00101 & .00045 & .00031 & .00030 & .00065 \\
1.1 & .00085 & .00035 & .00021 & .00021 & .00052 \\
1.2 & .00072 & .00028 & .00015 & .00016 & .00042 \\
1.3 & .00062 & .00022 & .00011 & .00012 & .00035 \\
1.4 & .00053 & .00018 & .00008 & .00009 & .00029 \\
1.5 & .00047 & .00015 & .00006 & .00007 & .00025 \\
1.6 & .00041 & .00012 & .00004 & .00006 & .00021 \\
1.7 & .00036 & .00010 & .00003 & .00005 & .00018 \\
1.8 & .00032 & .00009 & .00003 & .00004 & .00016 \\
1.9 & .00029 & .00007 & .00002 & .00003 & .00014 \\
2.0 & .00026 & .00006 & .00002 & .00003 & .00012 \\
\hline \hline
\end{tabular}

To make the results of this research comparable with those of the previous work [3], [4], we have used 2.0 as the scale factor here for the selected four moment feature functions.
Based on the analysis of Table I (A) and Table I (B), all feature sets for different types of Gegenbauer moments are shown in Table II.

TABLE II: The SELECTED Four GEGENBAUER MOMENT FEATURES For DIFFERENT $\lambda S$

\begin{tabular}{ccccc}
\hline \hline$\lambda$ & $f_{1}$ & $f_{2}$ & $f_{3}$ & $f_{4}$ \\
\hline 0.1 & $2 A_{11}$ & $2 A_{12}$ & $2 A_{21}$ & $2 A_{30}$ \\
0.2 & $2 A_{11}$ & $2 A_{12}$ & $2 A_{21}$ & $2 A_{30}$ \\
0.3 & $2 A_{11}$ & $2 A_{12}$ & $2 A_{21}$ & $2 A_{30}$ \\
0.4 & $2 A_{02}$ & $2 A_{12}$ & $2 A_{21}$ & $2 A_{30}$ \\
0.5 & $2 A_{02}$ & $2 A_{12}$ & $2 A_{20}$ & $2 A_{21}$ \\
0.6 & $2 A_{00}$ & $2 A_{02}$ & $2 A_{12}$ & $2 A_{20}$ \\
0.7 & $2 A_{00}$ & $2 A_{02}$ & $2 A_{20}$ & $2 A_{30}$ \\
0.8 & $2 A_{00}$ & $2 A_{02}$ & $2 A_{20}$ & $2 A_{30}$ \\
0.9 & $2 A_{00}$ & $2 A_{02}$ & $2 A_{20}$ & $2 A_{30}$ \\
1.0 & $2 A_{00}$ & $2 A_{02}$ & $2 A_{20}$ & $2 A_{30}$ \\
1.1 & $2 A_{00}$ & $2 A_{02}$ & $2 A_{10}$ & $2 A_{20}$ \\
1.2 & $2 A_{00}$ & $2 A_{02}$ & $2 A_{10}$ & $2 A_{20}$ \\
1.3 & $2 A_{00}$ & $2 A_{02}$ & $2 A_{10}$ & $2 A_{20}$ \\
1.4 & $2 A_{00}$ & $2 A_{01}$ & $2 A_{10}$ & $2 A_{20}$ \\
1.5 & $2 A_{00}$ & $2 A_{01}$ & $2 A_{10}$ & $2 A_{20}$ \\
1.6 & $2 A_{00}$ & $2 A_{01}$ & $2 A_{10}$ & $2 A_{20}$ \\
1.7 & $2 A_{00}$ & $2 A_{01}$ & $2 A_{10}$ & $2 A_{20}$ \\
1.8 & $2 A_{00}$ & $2 A_{01}$ & $2 A_{10}$ & $2 A_{20}$ \\
1.9 & $2 A_{00}$ & $2 A_{01}$ & $2 A_{10}$ & $2 A_{20}$ \\
2.0 & $2 A_{00}$ & $2 A_{01}$ & $2 A_{10}$ & $2 A_{20}$ \\
\hline \hline & & & &
\end{tabular}

To measure the distance between any pair of Chinese characters in our four dimensional Gegenbauer moment function features space, we adopt the square root of the mean-square distance:

$d_{i j}=\sqrt{\left(f_{1 i}-f_{1 j}\right)^{2}+\left(f_{2 i}-f_{2 j}\right)^{2}+\left(f_{3 i}-f_{3 j}\right)^{2}+\left(f_{4 i}-f_{4 j}\right)^{2}}$,

where $i$ and $j$ represent any two Chinese characters, $1 \leq i, j \leq 6,763$.

The results of applying different types of Gegenbauer moment features to the Chinese character set are shown in Table III and Table IV. Table III shows the numbers of Chinese character pairs within the mean-square distance of 0.1 for different $\lambda$ values between 0.1 and 0.5 , and Table IV displays the statistics on the Chinese character pairs for $\lambda$ values between 0.5 and 2.0. As mentioned above, when $\lambda=0.5$, the Gegenbauer moments become the Legendre moments [5]-[7].

The results presented in Table III and Table IV clearly show that, in terms of recognition power, the Gegenbauer moment descriptors with smaller $\lambda$ values are more powerful than those with larger $\lambda$ values. 
TABLE III: STATISTICS ON THE CHINESE CHARACTER PAIRS WITHIN DISTANCE 0.1

\begin{tabular}{cccccc}
\hline \hline$d_{i j} \leq$ & $\lambda=0.1$ & $\lambda=0.2$ & $\lambda=0.3$ & $\lambda=0.4$ & $\lambda=0.5$ \\
\hline 0.01 & 0 & 0 & 6 & 16 & 55 \\
0.02 & 0 & 4 & 35 & 165 & 616 \\
0.03 & 0 & 15 & 182 & 867 & 2,977 \\
0.04 & 0 & 42 & 589 & 2,752 & 9,620 \\
0.05 & 1 & 106 & 1,470 & 6,755 & 23,312 \\
0.06 & 3 & 219 & 3,048 & 13,700 & 47,598 \\
0.07 & 4 & 431 & 5,624 & 25,007 & 86,599 \\
0.08 & 7 & 722 & 9,434 & 42,140 & 144,154 \\
0.09 & 11 & 1,121 & 14,757 & 66,404 & 225,216 \\
0.1 & 15 & 1,680 & 22,266 & 99,969 & 334,275 \\
\hline \hline
\end{tabular}

TABLE IV: STATISTICS ON THE CHINESE CHARACTER PAIRS WITHIN

\begin{tabular}{ccccccc}
\multicolumn{7}{c}{ DisTANCE 0.01 } \\
\hline \hline$d_{i j} \leq$ & $\lambda=0.5$ & $\lambda=0.8$ & $\lambda=1.1$ & $\lambda=1.4$ & $\lambda=1.7$ & $\lambda=2.0$ \\
\hline 0.001 & 0 & 0 & 0 & 0 & 1 & 0 \\
0.002 & 0 & 0 & 1 & 2 & 5 & 6 \\
0.003 & 1 & 1 & 4 & 11 & 15 & 20 \\
0.004 & 2 & 5 & 20 & 23 & 42 & 68 \\
0.005 & 8 & 12 & 41 & 63 & 103 & 161 \\
0.006 & 11 & 32 & 80 & 134 & 219 & 312 \\
0.007 & 18 & 64 & 175 & 221 & 407 & 577 \\
0.008 & 26 & 110 & 276 & 411 & 687 & 977 \\
0.009 & 34 & 177 & 445 & 683 & 1,075 & 1,543 \\
0.01 & 55 & 265 & 655 & 1,043 & 1,650 & 2,352 \\
\hline \hline
\end{tabular}

TABLE V: CHINESE CHARACTER PAIRS WITH THE CLOSEST DISTANCE FOR

\begin{tabular}{|c|c|c|c|c|c|}
\hline \multicolumn{6}{|c|}{$\Lambda=0.1,0.2,0.3$} \\
\hline \multirow[t]{4}{*}{$\lambda=0.1$} & 烤苑 & 士 誊 & 䒾濑 & 憝綮 & 铃晌 \\
\hline & 0.04335 & 0.05454 & 0.05794 & 0.06734 & 0.07570 \\
\hline & 手庭 & 㴒娌 & 盟粗 & 疆 钜 & 师 䓠 \\
\hline & 0.07627 & 0.07953 & 0.08201 & 0.08383 & 0.08422 \\
\hline \multirow[t]{4}{*}{$\lambda=0.2$} & 徘誉 & 念猢 & 後炻 & 浸 遒 & 疠 裎 \\
\hline & 0.02493 & 0.02514 & 0.02623 & 0.02644 & 0.02690 \\
\hline & 岩鄢 & 砌 侵 & 弥 檩 & 恶 㐭 & 篇罧 \\
\hline & 0.02703 & 0.02703 & 0.02854 & 0.02971 & 0.02991 \\
\hline \multirow[t]{4}{*}{$\lambda=0.3$} & 傲跆 & 摇姍 & 荃掎 & 踌 珠 & 越绶 \\
\hline & 0.00863 & 0.00881 & 0.00881 & 0.00904 & 0.00921 \\
\hline & 臻燔 & 钵 愎 & 绷 温 & 借 廓 & 搬鼠 \\
\hline & 0.00981 & 0.01029 & 0.01131 & 0.01168 & 0.01187 \\
\hline
\end{tabular}

TABLE VI: THE SAME CHINESE CHARACTER PAIRS RECOGNIZED BY DIFFERENT $\Lambda$ VALUES

\begin{tabular}{|c|c|c|c|c|c|}
\hline & 烤苑 & " 士 誊 & "䒾濑 & 憝綮 & 铃晌 \\
\hline$\lambda=0.2$ & 0.21014 & 0.05092 & 0.31650 & 0.10049 & 0.12324 \\
\hline$\lambda=0.3$ & 0.20084 & 0.04312 & 0.27451 & 0.09478 & 0.12705 \\
\hline & 手庭 & 㴒娌 & 盟豢 & 疆 钜 & 师 䓠 \\
\hline$\lambda=0.2$ & 0.23747 & 0.28051 & 0.07465 & 0.18579 & 0.10824 \\
\hline$\lambda=0.3$ & $\begin{array}{c}0.21080 \\
\text { 徘誉 }\end{array}$ & $\begin{array}{c}0.25666 \\
\text { 念猢 }\end{array}$ & $\begin{array}{c}0.07404 \\
\text { 後炻 }\end{array}$ & $\begin{array}{c}0.17180 \\
\text { 浸遒 }\end{array}$ & $\begin{array}{c}0.08435 \\
\text { 疠 裎 }\end{array}$ \\
\hline$\lambda=0.1$ & 0.42208 & 0.25590 & 0.66929 & 0.33457 & 0.51769 \\
\hline$\lambda=0.3$ & $\begin{array}{c}0.05200 \\
\text { 岩鄢 }\end{array}$ & $\begin{array}{c}0.05693 \\
\text { 砌 侵 }\end{array}$ & $\begin{array}{c}0.09951 \\
\text { 弥檩 }\end{array}$ & $\begin{array}{c}0.05538 \\
\text { 恶兽 }\end{array}$ & $\begin{array}{c}0.05876 \\
\text { 篇罞 }\end{array}$ \\
\hline$\lambda=0.1$ & 0.30677 & 0.23785 & 0.53944 & 0.47703 & 0.77320 \\
\hline$\lambda=0.3$ & $\begin{array}{c}0.05574 \\
\text { 傲跆 }\end{array}$ & $\begin{array}{c}0.05178 \\
\text { 摇姍 }\end{array}$ & $\begin{array}{c}0.05836 \\
\text { 荃掎 }\end{array}$ & $\begin{array}{c}0.08045 \\
\text { 踌珠 }\end{array}$ & $\begin{array}{c}0.07729 \\
\text { 越绶 }\end{array}$ \\
\hline$\lambda=0.1$ & 1.13748 & 0.63457 & 0.95404 & 0.28392 & 0.40662 \\
\hline$\lambda=0.2$ & $\begin{array}{c}0.17525 \\
\text { 臻燔 }\end{array}$ & $\begin{array}{c}0.09824 \\
\text { 钵愎 }\end{array}$ & $\begin{array}{c}0.13577 \\
\text { 朋 温 }\end{array}$ & $\begin{array}{c}0.04066 \\
\text { 借廓 }\end{array}$ & $\begin{array}{c}0.08421 \\
\text { 搬菂 }\end{array}$ \\
\hline$\lambda=0.1$ & 1.23814 & 0.90709 & 0.53045 & 1.08458 & 0.49081 \\
\hline$\lambda=0.2$ & 0.17001 & & & 0.14433 & 0.07856 \\
\hline
\end{tabular}

Table VI shows that the same Chinese character pairs listed in Table V classified by the same set of Gegenbauer moment features with different $\lambda$ values. It is clearly shown that some Chinese character pairs are difficult to be recognized by using one $\lambda$ value, but is much easier to be classified by using other values. In other words, one object recognition system based on the Gegenbauer moment descriptors can perform as a group of recognition systems to the same set of objects by merely changing one parameter value.

TABLE VII: SOME CHINESE CHARACTER PAIRS WHICH ARE VERY CLOSE IN SHAPES AND ARE DiFFICULT TO RECOGNIZE FOR SOME EXISTING CHINESE

\begin{tabular}{|c|c|c|c|c|c|}
\hline \multicolumn{6}{|c|}{ OCR SYSTEMS } \\
\hline & "人入 & " 儿几 & "厂广 & 刀刃 & " 刀フ \\
\hline$\lambda=0.1$ & 2.44622 & 2.78736 & 5.25406 & 1.83271 & 5.31728 \\
\hline$\lambda=0.2$ & 0.69757 & 0.64991 & 1.30843 & 0.54733 & 1.50938 \\
\hline \multirow[t]{2}{*}{$\lambda=0.3$} & 0.34540 & 0.26688 & 0.57849 & 0.28658 & 0.74933 \\
\hline & 土土 & 下六 & 大太 & 大犬 & 千于 \\
\hline$\lambda=0.1$ & 3.49081 & 2.17933 & 3.38498 & 5.51028 & 4.71231 \\
\hline$\lambda=0.2$ & 0.97797 & 0.59513 & 0.97864 & 1.33205 & 1.27290 \\
\hline$\lambda=0.3$ & $\begin{array}{c}0.47323 \\
\text { 千干 }\end{array}$ & $\begin{array}{c}0.27991 \\
\text { 干于 }\end{array}$ & $\begin{array}{c}0.49635 \\
\text { 己已 }\end{array}$ & $\begin{array}{c}0.56600 \\
\text { 己巳 }\end{array}$ & $\begin{array}{c}0.60078 \\
\text { 尸户 }\end{array}$ \\
\hline$\lambda=0.1$ & 1.26565 & 3.62095 & 1.90407 & 3.07617 & 3.31135 \\
\hline$\lambda=0.2$ & 0.37232 & 0.93635 & 0.47409 & 0.81679 & 0.96650 \\
\hline \multirow[t]{2}{*}{$\lambda=0.3$} & 0.18793 & 0.42577 & 0.21689 & 0.39545 & 0.50361 \\
\hline & 天夭 & 木术 & 王玉 & 王主 & 夫失 \\
\hline$\lambda=0.1$ & 2.02222 & 0.72123 & 0.69226 & 2.48306 & 1.39324 \\
\hline$\lambda=0.2$ & 0.57861 & 0.29736 & 0.22959 & 0.63861 & 0.50353 \\
\hline \multirow[t]{2}{*}{$\lambda=0.3$} & 0.28757 & 0.18480 & 0.12718 & 0.28610 & 0.29868 \\
\hline & 日曰 & 万方 & 今令 & 井并 & 他池 \\
\hline$\lambda=0.1$ & 1.14534 & 1.70662 & 1.29174 & 2.43081 & 1.97371 \\
\hline$\lambda=0.2$ & 0.30140 & 0.45714 & 0.40958 & 0.68661 & 0.83529 \\
\hline \multirow{2}{*}{$\lambda=0.3$} & 0.13839 & 0.21827 & 0.22134 & 0.33730 & 0.53088 \\
\hline & 扑朴 & 未末 & 冊母 & 扬杨 & 观 现 \\
\hline$\lambda=0.1$ & 2.49502 & 0.76764 & 1.65907 & 3.50590 & 2.90213 \\
\hline$\lambda=0.2$ & 0.77840 & 0.21961 & 0.54503 & 0.97390 & 0.77516 \\
\hline \multirow[t]{2}{*}{$\lambda=0.3$} & 0.41078 & 0.10697 & 0.30262 & 0.46954 & 0.36366 \\
\hline & 现 规 & 听昕 & 如 知 & 论 沦 & 伦 抢 \\
\hline$\lambda=0.1$ & 2.38262 & 2.19267 & 4.86786 & 4.77826 & 1.75680 \\
\hline$\lambda=0.2$ & 0.57243 & 0.56937 & 1.36209 & 1.29040 & 0.50619 \\
\hline \multirow[t]{2}{*}{$\lambda=0.3$} & 0.25618 & 0.26577 & 0.66011 & 0.60711 & 0.24385 \\
\hline & 沙抄 & 垃拉 & 诗 待 & 拒柜 & 怕 帕 \\
\hline$\lambda=0.1$ & 1.75382 & 2.90987 & 1.39122 & 3.32366 & 0.81031 \\
\hline$\lambda=0.2$ & 0.77292 & 0.96118 & 0.52105 & 0.88007 & 0.17445 \\
\hline \multirow[t]{2}{*}{$\lambda=0.3$} & 0.48713 & 0.50712 & 0.28419 & 0.40757 & 0.12454 \\
\hline & 奖桨 & 俊 浚 & 茶茶 & 诽 排 & 借 惜 \\
\hline$\lambda=0.1$ & 5.22165 & 5.00748 & 1.70758 & 4.25970 & 2.16760 \\
\hline$\lambda=0.2$ & 1.47669 & 1.37398 & 0.42739 & 1.29275 & 0.67991 \\
\hline \multirow[t]{2}{*}{$\lambda=0.3$} & 0.71573 & 0.66697 & 0.18637 & 0.66318 & 0.37307 \\
\hline & 惜 错 & 粘 钻 & 捡 检 & 获获 & 竞 竟 \\
\hline$\lambda=0.1$ & 3.23254 & 1.86286 & 4.44990 & 5.29989 & 0.46041 \\
\hline$\lambda=0.2$ & 0.90988 & 0.43290 & 1.20614 & 1.39810 & 0.16558 \\
\hline \multirow[t]{2}{*}{$\lambda=0.3$} & 0.44861 & 0.17422 & 0.56756 & 0.65259 & 0.09809 \\
\hline & 倍培 & 维 锥 & 毫豪 & 婏 換 & 壕嚎 \\
\hline$\lambda=0.1$ & 2.87105 & 2.67289 & 5.13444 & 2.04071 & 7.62403 \\
\hline$\lambda=0.2$ & 0.88201 & 0.70870 & 1.59720 & 0.63399 & 2.05458 \\
\hline$\lambda=0.3$ & 0.45772 & 0.32617 & 0.82569 & 0.33369 & 0.95720 \\
\hline
\end{tabular}

Table V shows each of the closest ten pairs of Chinese characters in the four dimensional Gegenbauer moment descriptor space with different $\lambda$ values $0.1,0.2$, and 0.3 . Note that, even though the Gegenbauer moment feature sets shown in Table II are the same when $\lambda$ 's values are $0.1,0.2$, and 0.3 , there is no any Chinese character pair appearing twice in Table V. This further indicates that, in terms of object recognition, the same set of Gegenbauer moment features with different $\lambda$ values perform as the different types of moment descriptors.

By examining Table VI, it is worthy to note that all the Chinese character pairs that are close in the Gegenbauer 
moment feature space, regardless the $\lambda$ values, are not similar in terms of Chinese character specific features such as components and number of strokes. It means that all of those Chinese character pairs should be relatively easier to recognize with traditional Chinese character recognition methods that focus more on local properties such as components and strokes.

To view the performance of Gegenbauer moment descriptors on Chinese character pairs that are similar in terms of local structures, we use some Chinese character pairs that often have difficulty to be recognized by some existing Chinese Optical Character Recognition (OCR) systems [8], [9]. Table VII lists some similar Chinese character pairs and the distances in the 4-d Gegenbauer moment feature space. The results show that the moment descriptors perform well for all these pairs. It is a natural conclusion that the proposed object recognition system based on moment features is a good complement for other object recognition techniques based on local structure features.

\section{OBSERVATIONS AND CONCLUSIONS}

A new object recognition model with moment descriptors is proposed in this research. Based on our analysis, from statistics perspective, we select four lower order moments with the highest variance values to form a set of moment features in the four dimensional Gegenbauer moment space.

To test our new Gegenbauer moment features, we use the set of 6,763 Chinese characters defined in China's national standard GB2312, with the font of song, as the testing images The size of each Chinese character is $24 \times 24$, while all pixels representing a Chinese character have the value 0 and those of background have the value 1. Due to the fact that many Chinese characters are different in meanings but are very similar in shapes, the Chinese character recognition is considered as a challenging task in the field of object recognition.

The experimental results in this research show that the proposed moment features selection procedure performs very well. Compared with the previous works [3], [4], the new moment features in the four dimensional Gegenbauer moment space are able to provide much improved recognition power.

In other words, the new moment features are simpler in form, but are more effective in terms of object recognition.

Moments are global features apart from specific features of an object, therefore, they perform extremely on some specific object recognition systems. For example, one of the most difficult problems for Chinese character recognition systems is due to the complicated structures of Chinese characters. Our experimental results have shown that all testing Chinese character pairs that have the closest distances are not alike in shapes at all. This makes the moment description a desired complement for other Chinese character recognition techniques based on local structure features [8], [9].

Furthermore, for some Chinese character pairs that are difficult to be recognized with a set of Gegenbauer moment features, they can be easily classified by the same set of Gegenbauer moment features with a different parameter $\lambda$. This suggests that the same set of Gegenbauer moment features with different $\lambda$ values can work together to improve the recognition of rate of an object recognition system. From the implemental point of view, the Gegenbauer moment features are very efficient recognition features that can perform different types of moment descriptors within the same system.

Computing image moments with hardware has been one of the fronts in moment methods in recent years [10]. With the simplified moment function features proposed in this research, the lower order moment features of many different types moments can be designed and realized with hardware architectures with less difficulties.

\section{REFERENCES}

[1] M. K. Hu, "Visual problem recognition by moment invariant," IRE Trans. Inform. Theory, vol. IT-8, pp. 179-187, Feb. 1962.

[2] R. J. Prokop and A. P. Reeves, "A survey of moment-Based techniques for unoccluded object representation and recognition," Graphical Models and Image Processing, vol. 54, no. 5, pp. 438-460, 1992.

[3] S. Liao and Q. Lu, "A study of moment functions and its use in Chinese character recognition," in Proc. Fourth International Conference on Document Analysis and Recognition, August, 1997, pp. 572-575.

[4] S. Liao, A. Chiang, Q. Lu, and M. Pawlak, "Chinese character recognition via gegenbauer moments," in Proc. the 16th International Conference on Pattern Recognition, vol. 3, 2002, pp. 485-488.

[5] G. Szego, "Orthogonal polynomials," American Mathematical Society Providence, Rhode Island, 2000.

[6] G. Sansone, Orthogonal functions, New York: Dover Publications, Inc., 1991.

[7] S. X. Liao and M. Pawlak, "On image analysis by moments," IEEE Trans. PAMI, vol. 18, no. 3, pp. 254-266, 1996.

[8] Y. H. Zhang and C. P. Liu, "The analysis and suggestion to the evaluation of Chinese character recognition systems," in Proc. 1992 International Conference on Chinese Information Processing, 1992, pp. 407-412.

[9] L. Chin, "A survey on Chinese computing research," Hong Kong Computer Journal, vol. 9, no. 12, 1993.

[10] L. Kotoulas and I. Andreadis, "Efficient hardware architectures for computation of image moments," Real-Time Imaging, vol. 10, 371-378, 2004 .

Simon X. Liao received the BSc degree in 1982 from Beijing Normal University, Beijing, China, and the MSc and $\mathrm{PhD}$ degrees in electrical and computer engineering from the University of Manitoba, Winnipeg, Canada, in 1988 and 1993, respectively. Dr. Liao has been with the University of Winnipeg, Winnipeg, Canada, since 1991 . He is currently a professor in the Department of Applied Computer Science. His research interests include pattern recognition, computer vision, and image processing.

Jing Chen is an associate professor in the Department of Business and Administration, University of Winnipeg. Her research interests include statistical analysis, supply chain management, and pricing and revenue management. 